\title{
KLF15 is a protective regulatory factor of heart failure induced by pressure overload
}

\author{
SHU-FAN ZOU ${ }^{1}$, YANG YU ${ }^{1}$, YAN PENG $^{2}$, YING-BIN XIAO ${ }^{1}$, ZHAO JIAN $^{1}$, \\ YUN-HAN JIANG ${ }^{1}$, SAI CHEN ${ }^{1}$, FU-QIN TANG ${ }^{1}$ and LIN CHEN $^{1}$ \\ ${ }^{1}$ Department of Cardiovascular Surgery, Xinqiao Hospital Affiliated to Army Medical University, Chongqing 400030; \\ ${ }^{2}$ Department of Cardiology, Daping Hospital Affiliated to Army Medical University, Chongqing 400042, P.R. China
}

Received April 12, 2019; Accepted December 9, 2019

DOI: $10.3892 / \mathrm{mmr} .2020 .10913$

\begin{abstract}
The aim of the present study was to investigate the protective effect of Kruppel-like factor 15 (KLF15) overexpression on heart failure (HF) induced by left ventricular (LV) pressure overload in mice. Wild-type (WT) mice and cardiac-specific KLF15-overexpressed transgenic (TG) mice were selected as research subjects, and an LV pressure overload model was constructed by ascending aortic constriction surgery. Changes in cardiac morphology and function, and ultrastructure and molecular expression were observed via M-mode echocardiography, histological and immunohistochemical staining, ELISA and western blotting at 2 and 6 weeks of LV overload. WT and TG mice subjected to 2 weeks of overload displayed adaptive LV hypertrophy characterized by ventricular thickness, cardiomyocyte size, ejection fraction and fractional shortening of heart-lung weight ratio and KLF15, and increases in vascular endothelial growth factor (VEGF) expression without other pathological changes. WT mice subjected to 6 weeks of overload displayed enlargement of the LV chamber, severe interstitial remodeling, and HW/LW, cardiac capillary and heart function decline, accompanied by downregulated expression of KLF15 and VEGF, and upregulated expression of connective tissue growth factor, phosphorylated p38 (p-p38) and phosphorylated Smad3 (p-Smad3). In contrast, TG mice exhibited improved resistance to 6 weeks of overload and a slighter molecular expression response compared with WT mice. KLF15 was revealed to be a critical factor regulating the expression of CTGF, VEGF, p-p38 and p-Smad3, and could alleviate the progression from adaptive LV hypertrophy to decompensatory cardiac insufficiency.
\end{abstract}

Correspondence to: Dr Lin Chen, Department of Cardiovascular Surgery, Xinqiao Hospital Affiliated to Army Medical University, 183 Xinqiao, Shapingba, Chongqing 400030, P.R. China E-mail: 409425152@qq.com

Key words: Kruppel-like factor 15, pressure overload, interstitial remodeling, myocardial hypertrophy, transgenic mice

\section{Introduction}

Aortic valve stenosis is a common disease of left ventricular (LV) overload, resulting in heart failure (HF) and mortality. Surgery is considered as the main treatment in the unload process (1). The benefits of surgery can differ greatly between patients. In a proportion of patients who had a long course of disease, there was little improvement in heart function (2). Furthermore, these patients were subjected to higher perioperative risk and surgical mortality (3). Myocardial ultrastructural changes induced by LV pressure overload was deemed to be a major molecular event hindering cardiac function recovery. Therefore, it is essential to investigate the changes in myocardial ultrastructure over different periods of LV overload, as well as the underlying molecular mechanisms, in order to develop clinical treatments.

Previous studies have demonstrated that following an initial period of LV overload, adaptive left ventricular hypertrophy (LVH) maintained the cardiac output against an increasing resistance to ejection $(4,5)$, which was characterized by cardiomyocyte hypertrophy and ventricular wall thickening. Gradually, the adaptive LVH changed into cardiac insufficiency, and even heart failure (HF), if the condition of pressure overload continued (6). A large number of studies have used failing heart tissue to demonstrate pathological changes, including severe interstitial fibrosis and excessive collagen I and III deposition $(7,8)$. The heart function was hardly improved and was reversed at this stage, even if the pressure overload was relieved (9). A study by Oka et al (10) also demonstrated that the density of capillaries was greatly decreased, and that the microcirculation of myocardium was in a state of hypoxia. Therefore, the present study speculated that various pathological changes in the myocardial interstitium, rather than in the cardiomyocytes, may be the cause of irreversible impairment of cardiac function, however the molecular mechanism underlying the progression of adaptive $\mathrm{LVH}$ to HF induced by LV overload remains unclear.

Kruppel-like factor 15 (KLF15), a member of the Kruppel-like factors (KLFs), which are a subclass of the zinc-finger family of transcriptional regulators, is known to participate in a diverse range of biological processes, such as cell proliferation, differentiation, migration and apoptosis $(11,12)$. Over the past decade, research has focused on its 
vital protective effect in the cardiovascular field. It has previously been demonstrated that KLF15 is expressed in myocardial tissue and notably decreased in HF tissue, both in rodents and humans (13). A previous study also revealed that the expression of KLF15 was decreased in prolonged LV pressure overload in rats, and sustained low expression after unloading, accompanied by severe collagen deposition and interstitial fibrosis (9). Another study demonstrated that under conditions of aortic constriction, KLF15 null(-/-) mice lapsed into HF in a short time compared with wild-type (WT) mice (14). It was recently verified that KLF15 could stimulate the process of endothelial cell proliferation and transformation into tube-like structures in vitro (15). Overall, the expression level of KLF15 was negatively associated with the development of the HF process, and the present study speculated that KLF15 could protect heart function by means of inhibiting cardiac interstitial remodeling and promoting angiogensis. However, the precise underlying molecular mechanism remains unknown. The present study constructed a mouse model of ascending aortic constriction (AAC) (9) and included cardiac-specific KLF15-overexpressed transgenic mice to clarify the pathological progression and molecular mechanism underlying LV pressure overload.

\section{Materials and methods}

Cardiac-specific KLF15-overexpressed transgenic mice. The vector pRP.ExSi- $\alpha$ MHC-Klf15 was constructed and microinjected into the oosperm of C57BL/6J mice. Following surrogacy and PCR identification, two cardiac-specific KLF15-overexpressed transgenic (TG) founders were obtained. All the aforementioned processes were completed by Cyagen Biosciences, Inc. The founders were mated with wild-type (WT) mice to obtain F1 generation mice. Tail DNA was extracted for PCR detection by Premix Taq (Takara Bio). According to the protocol by Cyagen Biosciences, Inc., the forward primer for the transgene was 5'-AGAAGCAGGCACTTTACATGG-3', and the reverse primer was 5'-AGAAGCAGGCACTTTACATGG-3'. The transgene PCR product size was $333 \mathrm{bp}$. Total cardiac protein from the F1 generation of mice that was identified as positive was extracted in order to assess the expression of KLF15 via western blotting and for screening the KLF15-overexpressed mice in the following study (16).

LV pressure overload animal model establishment. All procedures abided by the Guide for the Care and Use of Laboratory Animals (Department of Health and Human Services publication no. NIH 78-23,1996) and were approved by the Committee on Animal Research of Army Military Medical University, Chongqing, China. Male C57BL/6J WT mice, 8-10-weeks of age, weighing 21.0-25.0 g, were provided by the Experimental Animal Center of Xinqiao Hospital, and cardiac-specific KLF15-overexpressed transgenic (TG) mice were provided by Cyagen Biosciences, Inc. The mice were used to construct the LV overload models by means of an AAC surgery without artificial ventilation. All mice were deprived of food and allowed a small amount of water $8 \mathrm{~h}$ before surgery.

The present study used $1.2 \%$ tribromoethyl alcohol $(0.24 \mathrm{mg} / \mathrm{g})$ as an anesthetic by intraperitoneal injection and fixed the overturned mice. A transverse skin incision was then made in the suprasternal fossa after shaving and sterilizing.
The sternum was cut lengthwise to the level of the second rib and the soft tissue was blunt dissected from the superficial trachea. The upper ascending aorta was exposed after the separation of the thymus, 4-0 sutures encircled the ascending aorta and a 27-gauge needle was placed parallel to it. The needle was withdrawn rapidly after binding. The aforementioned procedure was performed with care in order to avoid damage to pleura and causing pneumothorax. The diameter of the ascending aorta dropped to $\sim 50 \%$ after banding and the incision was closed when the respiration and heartbeat were stable. Following surgery, the mice were allowed to eat and drink freely. A proportion of mice underwent a sham operation as a negative control.

The mice were divided into eight groups as follows: i) WT mice 2-weeks sham group (WT sham 2w); ii) WT mice 6-weeks sham group (WT sham6w); iii) TG mice 2-weeks sham group (TG sham2w); iv) TG mice 6-weeks sham group (TG sham6w); v) WT mice 2-weeks aortic banding (WT AB2w); vi) WT mice 6-weeks aortic banding (WT AB6w); vii) TG mice 2 -weeks aortic banding (TG AB2w); viii) TG mice 6-weeks aortic banding (TG AB6w).

Echocardiographic measurements of heart morphology and function. After being anesthetized by $1.2 \%$ tribromoethyl alcohol intraperitoneal injection, each group of mice was assessed using the M-mode echocardiography of VEVO 2100 Imaging system to measure heart rate (HR), systolic left ventricular posterior wall thickness (LVPWs), diastolic left ventricular posterior wall thickness (LVPWd), systolic interventricular septal thickness (IVSs), diastolic interventricular septal thickness (IVSd), left ventricular end systolic inside diameter (LVIDs), left ventricular end diastolic inside diameter (LVIDd), ejection fraction (EF) and fractional shortening (FS) 2 and 6 weeks after surgery.

Heart-lung weight ratio measurement. Each group of mice was anesthetized by $4 \%$ isoflurane inhalation and decapitated. The hearts and lungs were removed at the corresponding time. The attached adipose and vascular tissue was excised and residual blood was washed away with phosphate-buffered saline (PBS). The heart weight (HW) and lung weight (LW) were measured using precision electronic scales (Sartorius, Inc.) after drying them gently with sterile gauze and the HW/LW ratio was calculated.

Histological analysis of heart microstructural changes. Myocardial tissue was preserved with polyformaldehyde and cryopreservation. Each specimen was sectioned to a $5-\mu \mathrm{m}$ thickness after embedding in paraffin. Hematoxylin and eosin (H\&E) (Beijing Solarbio Science \& Technology Co., Ltd.) staining and Masson staining (Beijing Solarbio Science \& Technology Co., Ltd.) were used to assess the myocardial morphology and level of fibrosis. For Masson staining, the paraffin sections were deparaffinized, washed, and stained with hematoxylin staining solution for 5-10 min at room temperature. Next, the sections were stained with Masson Ponceau acid fuchsin solution for 5-10 min at room temperature, followed by immersion cleaning in $2 \%$ glacial acetic acid aqueous solution then differentiation with $1 \%$ phosphomolybdic acid aqueous solution for 3-5 min, and staining 
A

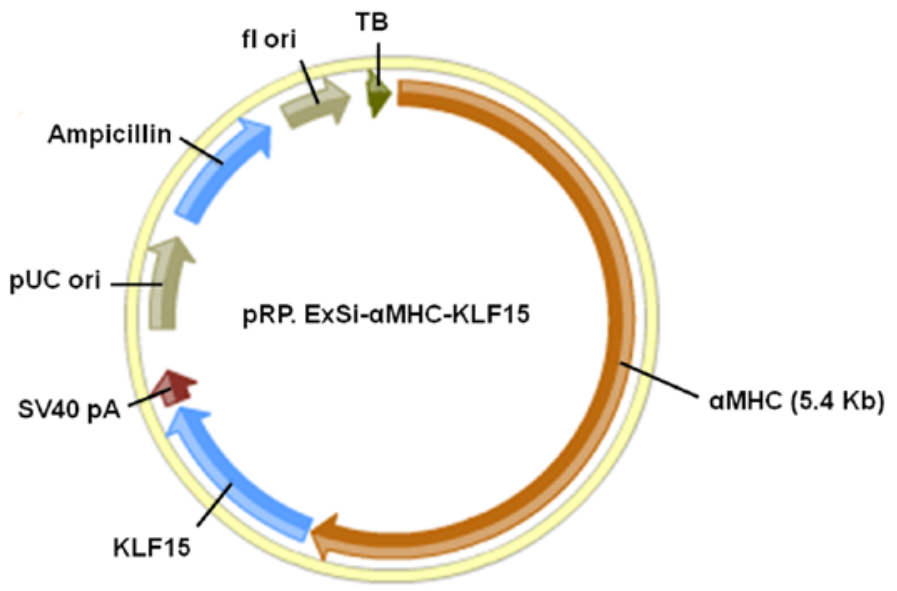

B

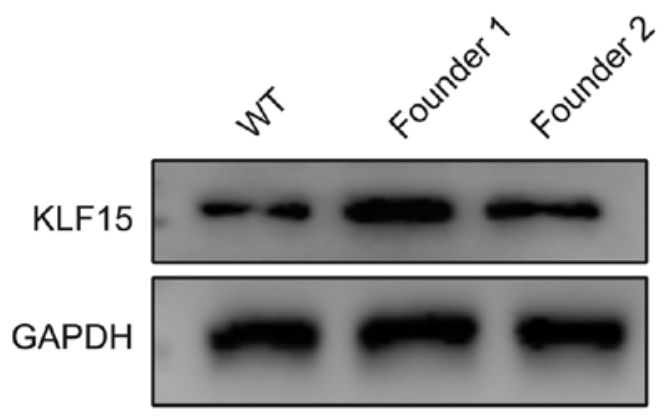

C

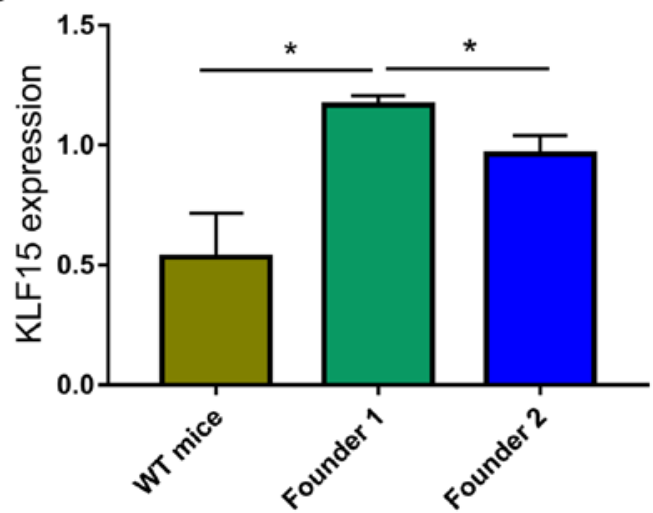

Figure 1. Establishment and identification of cardiac-specific KLF15 overexpression in transgenic mice. (A) Construction of the vector pRP.ExSi- $\alpha M H C-K 1 f 15$. (B) The specific expression of KLF15 factor in myocardial tissue was determined by western blotting. (C) Quantitative analysis of the expression of KLF15 factor in myocardial tissue. "P<0.05. KLF15, Kruppel-like factor 15.

with aniline blue or light green solution for $5 \mathrm{~min}$. Finally, the sections were mounted in neutral gum. For H\&E staining, the paraffin sections were dewaxed twice in xylene (10 min each). Sections were rehydrated sequentially in descending series of alcohol for 5 min each in anhydrous, 90, 80 and 70\% alcohol. Sections were then treated with phosphate buffered saline for 2 min. Specimens were stained by immersing in hematoxylin for $10 \mathrm{~min}$ at room temperature, treated with $1 \%$ acid alcohol for $3 \mathrm{sec}$, washed with running water for $10 \mathrm{~min}$, washed with distilled water for $2 \mathrm{~min}$, stained with $0.5 \%$ eosin for $3 \mathrm{~min}$ and washed with distilled water for $2 \mathrm{sec}$. Specimens were then dehydrated twice in $95 \%$ ethanol for 2 min each and cleared by treating twice with xylene for 5 min each. Sections were then mounted in neutral balsam. The specific marker CD31 on vascular endothelial cells (VECs) was exposed by immunohistochemical (IHC) staining to assess myocardial vascular density and distribution (17). The changes were collected and observed using a Leica DMIRB light microscope.

ELISA for assessment of collagen I and III deposition. The myocardial tissue of each group was ground to homogenate and the protein was extracted. Collagen type I and III were analyzed using an ELISA kit (FANKEWEI Bioscience, Inc.) according to the manufacturer's protocol, and statistical analyses were performed by comparing the standard curve.
KLF15,CTGF and VEGF expression levels and Smad 3 and 38 phosphorylation levels detected via western blotting. Western blotting was performed to identify the differential expression levels of KLF15, CTGF, VEGF, p-p38, t-p38, p-Smad3 and $\mathrm{t}-\mathrm{Smad} 3$ in each group. Proteins were extracted from an appropriate amount of ground frozen heart tissue by Cell Extraction Buffer (Thermo Fisher Scientific, Inc.) according to the manufacturer's instructions. The protein concentrations of the samples were measured using the BCA method. SDS-PAGE buffer was added to the samples at a unified dilution, boiled for $8 \mathrm{~min}$ and stored at $4^{\circ} \mathrm{C}$. SDS-PAGE $(10 \%)$ was performed on $20 \mu \mathrm{g}$ of each sample at $95 \mathrm{~V}$ for $120 \mathrm{~min}$, followed by electrophoretic transfer to a polyvinylidene difluoride membrane at $15 \mathrm{~V}$ for $50 \mathrm{~min}$. The bands were incubated at $4^{\circ} \mathrm{C}$ for $2 \mathrm{~h}$. The samples were then incubated with rabbit anti-mouse KLF15 (1:1,000, AV32587, Sigma-Aldrich; Merck KGaA), CTGF (1:1,000, ab6992, Abcam), VEGF (1:2,000, ab46154, Abcam), p-p38 (1:1,000, ab4822, Abcam), t-p38 (1:1,000, ab170099, Abcam), p-Smad3 (1:2,000, ab52903, Abcam), t-Smad3 (1:1,000, ab40854, Abcam) and GAPDH (1:10,000, ab181602, Abcam) antibodies at $4^{\circ} \mathrm{C}$ overnight. The following day, the samples were washed with PBST three times, followed by the addition of horseradish peroxidase-labeled goat anti-rabbit IgG (1:10,000, 31460 Invitrogen; Thermo Fisher Scientific, Inc.) and incubated at room temperature for $1 \mathrm{~h}$. The samples 
Table I. Heart weight, lung weight and heart weight/lung weight ratio at 2 weeks after aortic banding.

\begin{tabular}{|c|c|c|c|c|}
\hline \multirow[b]{2}{*}{ Parameter } & \multicolumn{2}{|c|}{ Sham } & \multicolumn{2}{|c|}{$\mathrm{AB}$} \\
\hline & WT $(n=5)$ & TG $(n=5)$ & WT $(n=5)$ & TG $(n=5)$ \\
\hline HW (mg) & $113.2 \pm 4.009$ & $112.2 \pm 5.128$ & $129.8 \pm 3.114^{\mathrm{a}}$ & $128.9 \pm 3.794^{a}$ \\
\hline LW (mg) & $136.5 \pm 2.368$ & $134.1 \pm 2.183$ & $133.9 \pm 3.270$ & $135.1 \pm 1.497$ \\
\hline $\mathrm{HW} / \mathrm{LW}(\mathrm{mg} / \mathrm{mg})$ & $0.831 \pm 0.035$ & $0.835 \pm 0.026$ & $0.973 \pm 0.041^{\mathrm{a}}$ & $0.954 \pm 0.022^{\mathrm{a}}$ \\
\hline
\end{tabular}

${ }^{\mathrm{a} P}<0.05$ vs. the corresponding sham. Data are represented as the mean \pm SEM. Sham, sham-operated; AB, aortic banding; WT, wild-type mice; TG, transgenic mice; HW, heart weight; LW, lung weight; HW/LW, heart-lung weight ratio.

Table II. The heart weight, lung weight and heart weight/lung weight ratio at 6 weeks after aortic banding.

\begin{tabular}{|c|c|c|c|c|}
\hline \multirow[b]{2}{*}{ Parameter } & \multicolumn{2}{|c|}{ Sham } & \multicolumn{2}{|c|}{$\mathrm{AB}$} \\
\hline & $\mathrm{WT}(\mathrm{n}=5)$ & TG $(n=5)$ & WT (n=5) & $\mathrm{TG}(\mathrm{n}=5)$ \\
\hline HW (mg) & $121.2 \pm 4.028$ & $120.9 \pm 4.574$ & $131.9 \pm 1.375^{\mathrm{a}}$ & $128.0 \pm 2.975$ \\
\hline LW (mg) & $144.1 \pm 2.357$ & $141.8 \pm 3.644$ & $194.8 \pm 2.159^{\mathrm{b}}$ & $167.1 \pm 2.768^{b, c}$ \\
\hline $\mathrm{HW} / \mathrm{LW}(\mathrm{mg} / \mathrm{mg})$ & $0.843 \pm 0.041$ & $0.852 \pm 0.020$ & $0.678 \pm 0.010^{\mathrm{b}}$ & $0.768 \pm 0.025^{\mathrm{a}, \mathrm{c}}$ \\
\hline
\end{tabular}

${ }^{\mathrm{a}} \mathrm{P}<0.05$ and ${ }^{\mathrm{b}} \mathrm{P}<0.01$ vs. the corresponding sham. ${ }^{\mathrm{c}} \mathrm{P}<0.05$ vs. WT $\mathrm{AB}$. Data are represented as the mean $\pm \mathrm{SEM}$. $\mathrm{AB}$, aortic banding; WT, wild-type mice; TG, transgenic mice; HW, heart weight; LW, lung weight; HW/LW, heart-lung weight ratio.

were rinsed again with PBST three times, stained with ECL reagent (Thermo Fisher Scientific, Inc.), and imaged with a Bio-Rad gel imaging system (Bio-Rad Laboratories). Quantity One software was used to analyze the gray value of the bands using GAPDH as the reference.

Statistical analysis. Experimental data were presented as the mean \pm standard error. The statistical difference was compared in each group using a one-way ANOVA, followed by a post hoc Tukey's test. All tests were performed using SPSS software (version 13.0; SPSS, Inc.) and GraphPad Prism software (version 7.0; GraphPad Software, Inc.). P<0.05 was considered to indicate a statistically significant difference.

\section{Results}

Cardiac expression of KLF15 is increased in TG mice compared with WT mice. The construction of the vector pRP. ExSi- $\alpha$ MHC-Klf15 (Fig. 1A) and integration of target genes was completed by Cyagen Biosciences, Inc. Ultimately, the present study obtained two TG founders, named founder 1 and founder 2. After mating with WT mice and identifying the F1 generation through tail DNA using PCR, 4 and 5 mice were identified as positive from the founder 1 and founder 2 mice, respectively. The present study then extracted total heart protein and examined the expression levels of KLF15 factor in myocardial tissue using western blot analysis, and quantified the average gray value with WT mice as a control. The results revealed that a basic expression level of KLF15 existed in WT mice, and the expression of KLF15 in the F1 generation of founder 1 was higher than in the F1 generation of founder 2 and WT mice (Fig. 1B). The difference was statistically significant $(\mathrm{P}<0.05$; Fig. $1 \mathrm{C})$. Founder 1 and its F1 generation were identified as positive and considered a suitable strain of KLF15 overexpression in the following study.

Mice survival. During the AAC surgery, the total mortality rate was $\sim 16.7 \%$. The main causes of death were pneumothorax $(\sim 4.2 \%)$, massive hemorrhage $(\sim 8.3 \%)$ and acute heart failure $(\sim 4.2 \%)$. The mortality rate of the sham operation was 0 . All mice in each group survived to the corresponding time-point after surgery, indicating that the degree of constriction was appropriate.

TG mice have delayed LV diameter increase and heart function decline. M-mode echocardiography is a dynamic curve image formed by light spot groups of the heart movement via mono beam scanning. IVS, LVID and LVPW are displayed from top to bottom. Fig. 2A demonstrates that the wave amplitude and ventricular wall thickness of WT AB2w and TG AB2w groups were markedly increased compared with the sham groups. In addition, AB2w induced a visible decrease in the LV chamber at the systolic period. Conversely, AB6w groups displayed an eccentric structural change featuring a weak amplitude and an enlargement of the LV chamber that was more evident in WT mice than in TG mice.

In addition, the present study assessed the HR, IVS, LVID, LVPW, EF and FS of each group for statistical analysis. As presented in Fig. 2B, the parameters did not differ between the WT sham and TG sham mice. At 2 weeks after aortic banding, the ventricular wall was significantly thicker and 
A

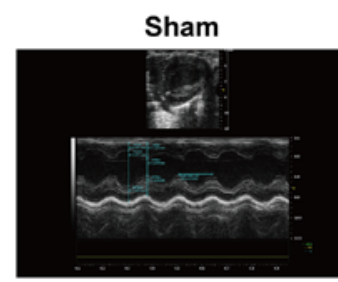

TG
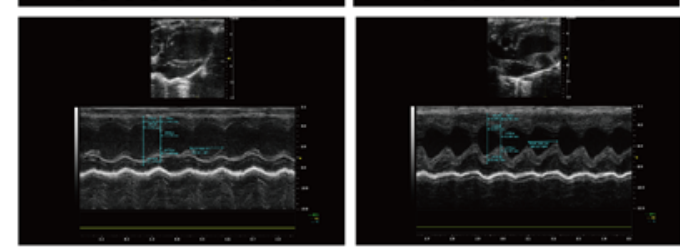

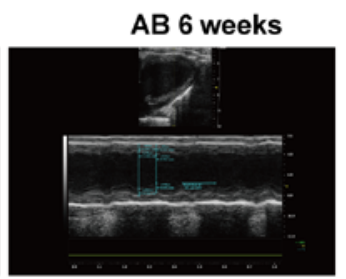

B
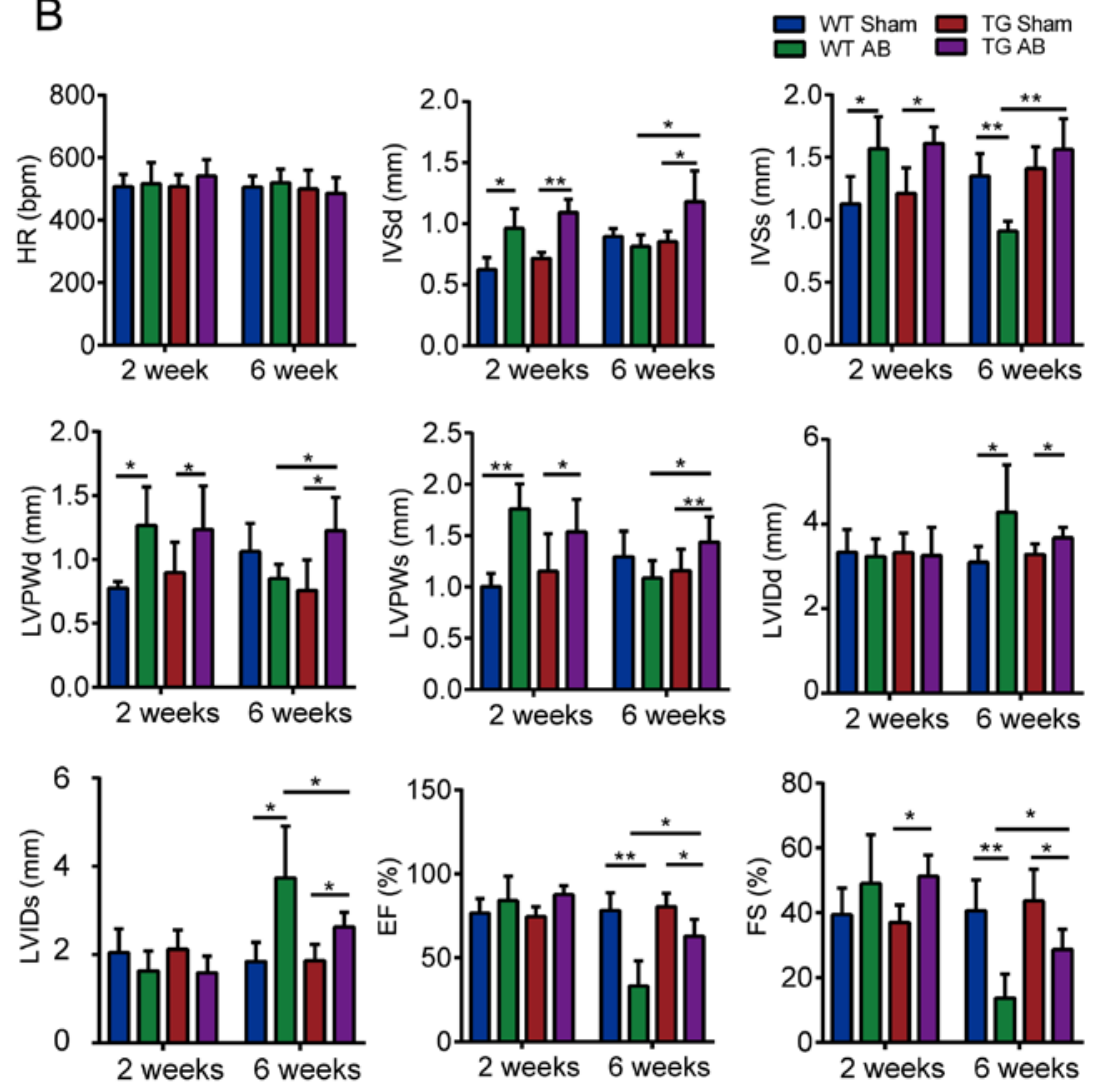

Figure 2. The effects of KLF15 on cardiac morphology and function measured by M-mode echocardiography. (A) M-mode echocardiography images via long axial section of the left ventricle. (B) Statistical results of the HR, IVSd, IVSs, LVPWd, LVPWs, LVIDd, LVIDs, EF, and FS of indicated groups ( $\mathrm{n}=5$ mice per group) by M-mode echocardiography of VEVO 2100 Imaging system. Data are represented as the mean $\pm \mathrm{SEM}$. *P<0.05; ${ }^{* *} \mathrm{P}<0.01$. Kruppel-like factor $15 ; \mathrm{HR}$, heart rate; IVSd, diastolic interventricular septal thickness; IVSs, systolic interventricular septal thickness; LVPWd, diastolic left ventricular posterior wall thickness; LVPWs, systolic left ventricular posterior wall thickness; LVIDd, left ventricular end diastolic inside diameter; LVIDs, left ventricular end systolic inside diameter; EF, ejection fraction; FS, fractional shortening; WT, wild-type; TG; transgenic; AB, aortic banding.

heart function compensation had increased in the AB groups compared with those in the corresponding sham groups $(\mathrm{P}<0.05)$, however the chamber diameter exhibited no significant difference. At 6 weeks after aortic banding, WT mice rather than TG mice demonstrated a significant decrease of $\mathrm{EF}$ and FS $(\mathrm{P}<0.01)$ and an increase of LVID compared with those in the sham groups $(\mathrm{P}<0.05)$. HR did not differ among the groups $(\mathrm{P}>0.05)$.

TG mice alleviate the $H W / L W$ decline induced by aortic banding for 6 weeks. Compared with the sham groups, the LW of WT mice and TG mice did not significantly change under LV overload conditions for 2 weeks, however, the HW and HW/LW increased. There was no significant difference between the WT mice and TG mice in the sham and AB groups, respectively (Table I). However, the LW of WT mice and TG mice with LV overload for 6 weeks was significantly higher and the HW/LW was lower than that of the sham mice, WT mice had a higher degree of change compared with TG mice. There was no significant difference in HW among groups (Table II).

Milder pathological changes in the myocardial interstitium of TG mice 6 weeks after aortic banding. $\mathrm{H} \& \mathrm{E}$ staining of myocardial sections in WT $\mathrm{AB} 2 \mathrm{w}$ and $\mathrm{TG} \mathrm{AB} 2 \mathrm{w}$ groups revealed 
A
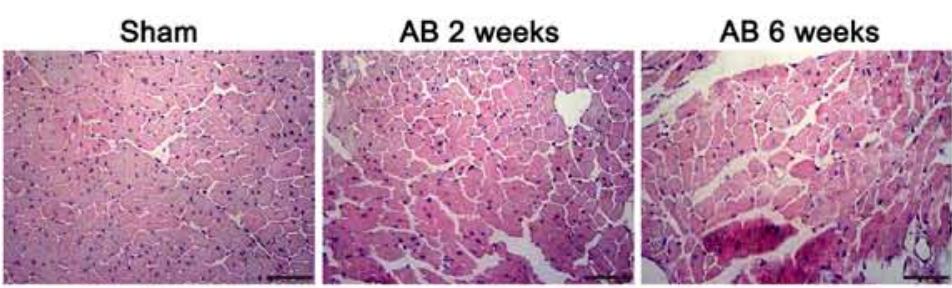

TG
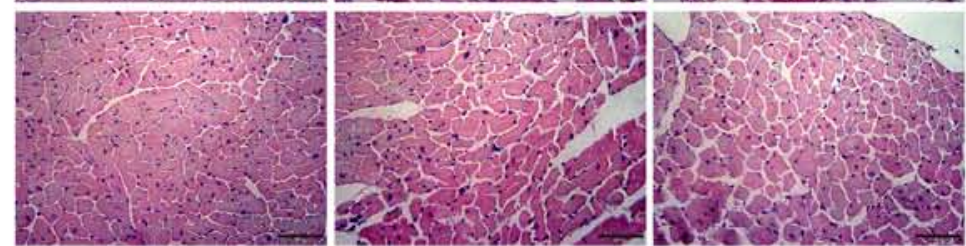

B

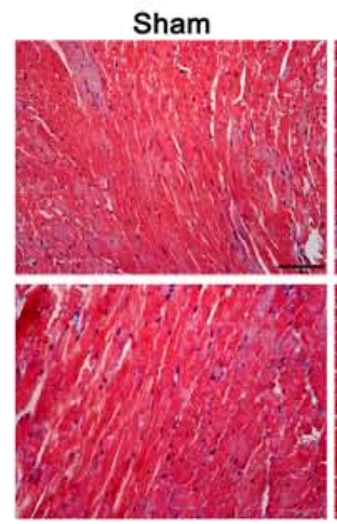

AB 2 weeks

AB 6 weeks
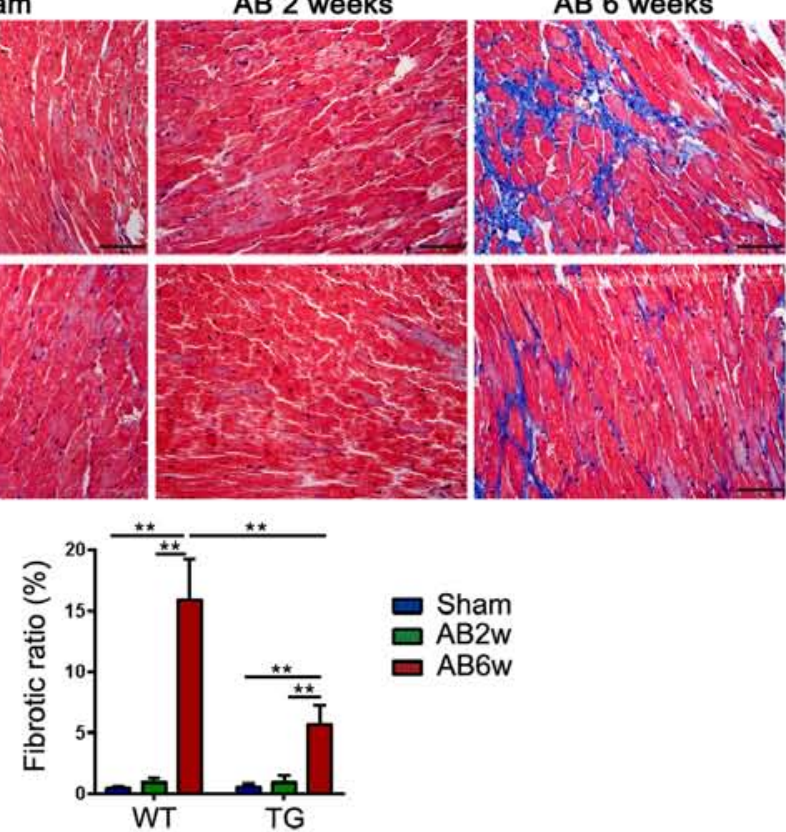

C
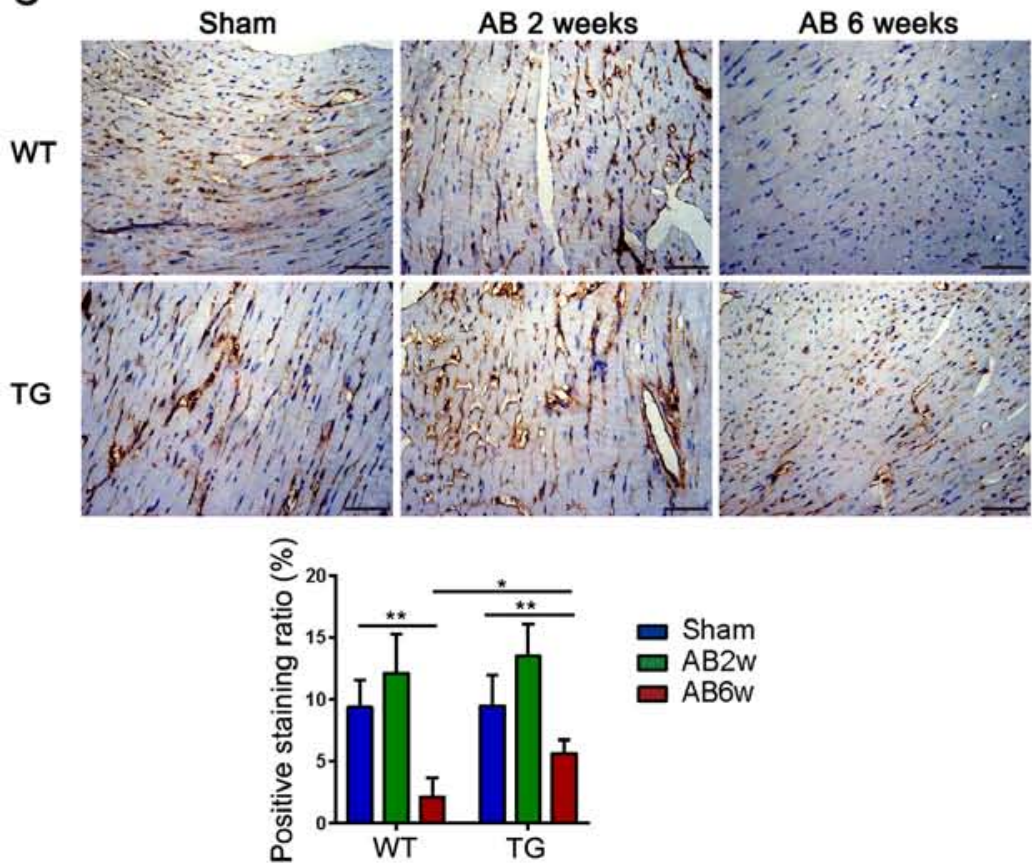

Figure 3. Myocardial ultrastructural changes induced by pressure overload and KLF15 overexpression. (A) H\&E staining of myocardial tissue sections in each group. Scale bar $=50 \mu \mathrm{m}$. (B) Masson staining of myocardial tissue sections in each group and quantitative measurement of fibrotic area ratio. Scale bar=50 $\mu \mathrm{m}$. (C) IHC staining of the specific marker CD31 on VECs and quantitative measurement of positive staining ratio. Scale bar $=50 \mu \mathrm{m}$. "P $<0.05$; ${ }^{* *} \mathrm{P}<0.01$. Kruppel-like factor 15; VECs, vascular endothelial cells; WT, wild-type; TG; transgenic; AB, aortic banding; AB2w; 2-week aortic banding; AB6w, 6-week aortic banding. 

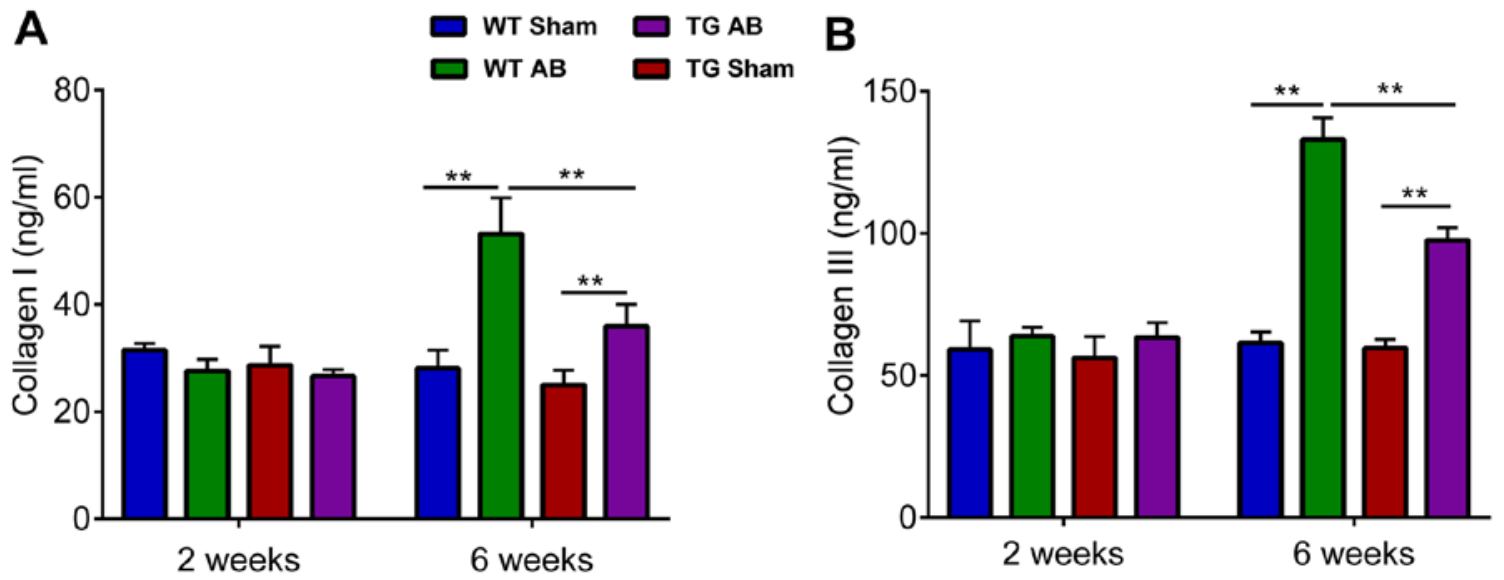

Figure 4. Content of collagen I and collagen III assessed by ELISA. (A) Content of collagen I in each group assessed by ELISA compared to the standard curve. (B) Content of collagen III in each group assessed by ELISA compared to the standard curve. Data are represented as the mean \pm SEM ( $\mathrm{n}=5$ ). ${ }^{* *} \mathrm{P}<0.01$. WT, wild-type; TG; transgenic; AB, aortic banding.

increased cardiomyocyte size and larger nuclei compared with the sham groups, and the cells were arranged regularly in both the sham and AB2w groups. Myocardial sections of mice subjected to aortic banding for 6 weeks (WT AB6w and TG AB6w) exhibited a shrinkage and irregular arrangement of cardiomyocytes compared with $\mathrm{AB} 2 \mathrm{w}$ groups, and this was more evident in WT mice than in TG mice (Fig. 3A). Masson staining revealed that aortic banding after 2 weeks did not induce cardiac interstitial fibrosis in either WT or TG mice. WT mice appeared to have severe fibrosis distributed in the myocardial interstitium primarily around the blood vessels compared with TG mice at 6 weeks after aortic banding, which was statistically significant $(\mathrm{P}<0.01$; Fig. 3B). As a specific marker for VECs, CD31 was demonstrated in brown via IHC staining. In WT and TG mice, the density was decreased in the AB6w groups compared with the sham groups $(\mathrm{P}<0.01)$. In addition, the TG AB6w group exhibited a higher density of CD31 than the WT AB6w group $(\mathrm{P}<0.05)$. No significant difference was observed between the sham groups and $\mathrm{AB} 2 \mathrm{w}$ groups $(\mathrm{P}>0.05$; Fig. $3 \mathrm{C})$.

Decreased collagen deposition in TG mice is induced by aortic banding for 6 weeks. When compared with the standard curve for quantification, the content of collagen I and III in myocardial tissue assessed by ELISA tended to coincide with the fibrosis demonstrated by Masson staining. Collagen I and III levels of TG and WT AB6w groups were increased compared to their corresponding sham groups $(\mathrm{P}<0.01$,) and there was also a statistically significant difference observed between these two groups $(\mathrm{P}<0.01)$ (Fig. 4). No significant difference was observed among the sham groups.

TG mice suppress CTGF upregulation and VEGF downregulation mediated by $p$-Smad 3 and $p$ - $p 38$. The present study assessed the expression levels of KLF15, CTGF, VEGF, Smad3 and p38 phosphorylation in each group using western blot analysis, and quantified the expression using the average gray value to investigate the role of each protein in the pathological progression of LV overload. The results revealed that under 2 weeks overload, the expression levels of CTGF and p-p38 were low and there was no significant difference between each group. The expression of VEGF and p-Smad3 were increased in the WT $\mathrm{AB} 2 \mathrm{w}$ and $\mathrm{TG} \mathrm{AB} 2 \mathrm{w}$ groups compared with the corresponding sham groups, but there was no statistically significant difference between the two groups. Compared with the WT sham 2w group, the expression of KLF15 was upregulated in the TG sham $2 \mathrm{w}, \mathrm{WT}$ AB2 $\mathrm{w}$ and TG AB2w groups, but there was no statistically significant difference among the three groups.

Under 6 weeks overload, the expression of p-p38 and p-Smad3 were upregulated in the WT AB6w and TG AB6w groups compared with the corresponding sham groups, but there was no statistically significant difference between the two groups. Furthermore, AB induced increased expression of CTGF and decreased expression of VEGF compared with the corresponding sham groups, which was even more evident in WT mice than in TG mice. The expression of KLF15 was downregulated in the WT AB6w group compared with the sham and TG AB6w groups (Fig. 5B).

\section{Discussion}

The present study demonstrated the histological and molecular expression changes in the pathological development process of LV overload in mice. An AAC operation was used instead of traverse aortic constriction (TAC) operation, which is regarded as a classic way to construct the ventricular hypertrophy model (18) to simulate the pathological process of LV pressure overload. The results of the present study demonstrated that in WT mice subjected to aortic banding for 2 weeks (WT AB2w), ventricular thickness, heart weight and capillary density increased, and LV systolic diameter decreased for enhancing contractility, EF, FS and KLF15 expression increased without other pathological changes. Gradually, the adaptive hypertrophy was turning into decompensation. At 6 weeks of aortic banding, WT mice exhibited a notable increase in LV diameter and lung weight, and a decrease in EF and FS, with an irregular arrangement of cardiomyocytes, severe interstitial fibrosis, collagen deposition and capillary density decline, accompanied by downregulated expression of KLF15 and VEGF, and upregulated expression of CTGF, p-p38 and p-Smad3. Notably, TG mice exhibited an improved level of resistance 

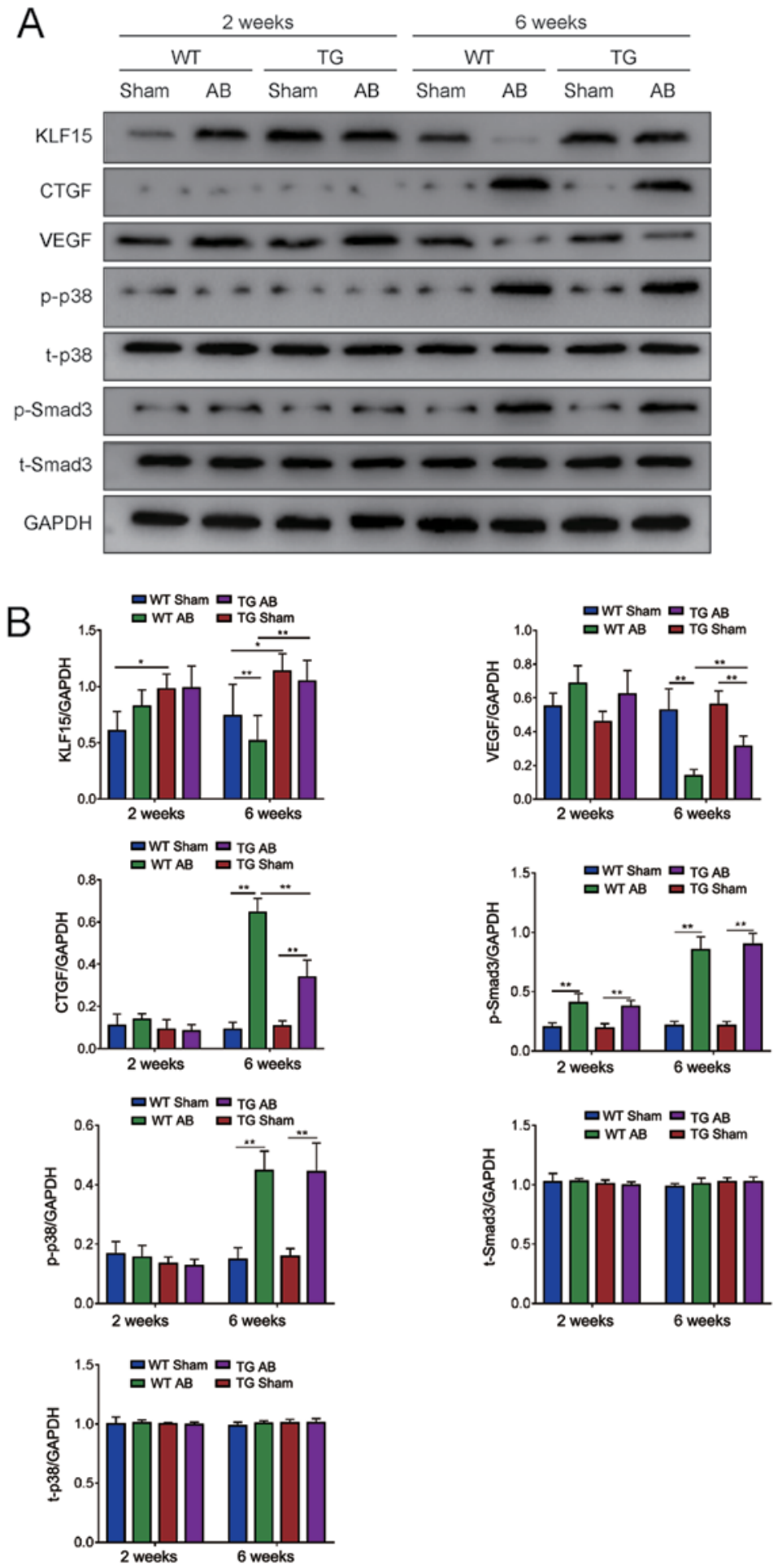

Figure 5. KLF15, CTGF, VEGF expression levels and Smad3, P38 phosphorylation levels in myocardial tissue detected by western blot analysis. (A) KLF15, CTGF, VEGF, p-Smad3, t-Smad3, p-p38, t-p38 expression in each group. (B) Quantitative measurement of KLF15, CTGF, VEGF, p-Smad3, t-Smad3, p-p38, t-p38 expression levels in each group. ${ }^{*} \mathrm{P}<0.05 ;{ }^{* *} \mathrm{P}<0.01$. Kruppel-like factor 15 ; VECs, vascular endothelial cells; VEGF, vascular endothelial growth factor; p-p38, phosphorylated p-38; p-Smad3, phosphorylated Smad3; WT, wild-type; TG; transgenic; AB, aortic banding.

than WT mice to prolonged LV pressure overload, and alleviated LV chamber enlargement, interstitial fibrosis, collagen deposition and capillary density decrease, and improved heart function compared with the WT mice. This model demonstrated different changes in compensatory and decompensatory stages at two time-points, including cardiac morphology, ultrastructure and molecular expression.
Compared with the classical TAC model, the AAC model could highlight the effect of mechanical pressure on the development of ventricular hypertrophy and decrease the interference of neurohumoral factors induced by increasing blood pressure in the brain. In addition, the whole procedure avoided endotracheal intubation and artificial ventilation, and increased the efficiency of model construction. This model 
effectively revealed myocardial interstitial remodeling rather than enlargement of individual cardiomyocytes associated with heart function decline, and non-cardiomyocytes in the heart played a significant role in the development process from the compensatory period to the decompensatory period. In fact, non-cardiomyocytes account for $\sim 70 \%$ of the total cell number in cardiac tissue, with the majority being fibroblasts and endotheliocytes (19). Previous studies have confirmed that under prolonged mechanical stimulation, cardiac fibroblasts transform into myofibroblasts participating in collagen secretion, elastin synthesis and interstitial fibrosis (20). Excessive collagen deposition and formation of collagen fibers resulting in extracellular matrix (ECM) remodeling promote LV inflexibility and the compressive deformation of cardiomyocytes contribute to cardiac insufficiency (21). Meanwhile, anatomic remodeling leads to electrophysiological changes and aggravated heart function (22). Furthermore, increased myocardial oxygen consumption, diffusion distance and capillary constriction caused by pressure overload place the myocardium in a state of microcirculation hypoxia and contractile dysfunction (23). Angiogenesis, as a vital physiological and pathological process in the inflammatory response and oncology (24), may be a therapeutic strategy towards HF recovery. In the present study, the density of vascular endotheliocyte specific marker CD31 in each group was positively associated with the corresponding EF and FS values. The proliferation and migration of endotheliocytes involved in angiogenesis display an effective compensation to a certain degree.

KLF15 has been demonstrated to be a key factor in cardiac remodeling $(25,26)$. A previous study demonstrated that under LV pressure overload, activated Smad3 promoted the expression of downstream CTGF, which was considered to be a classic pathway in the initiation of interstitial remodeling (27). Other studies have demonstrated that prolonged aortic constriction in rats could activate p38 mitogen-activated protein kinase (p38-MAPK) in cardiomyocytes, and would remain in sustainable activation even after LV pressure unload (25). The present study also revealed several new findings associated with KLF15. For one, the expression of KLF15 was revealed to be negatively associated with myocardial interstitial remodeling, rather than adaptive LVH. Under 2 weeks of overload, a compensatory increase of KLF15 expression inhibited p-Smad3/CTGF pathway and promoted angiogenesis by positively regulating VEGF expression, which may be a critical mechanism in repressing interstitial remodeling and maintaining heart function. In addition, the activation of p38 induced by LV pressure overload for 6 weeks could suppress the expression of KLF15, and low expression of KLF15 lost its ability to inhibit CTGF expression and angiogenesis by regulating VEGF expression, resulting in occurrence of myocardial interstitial remodeling and HF. Furthermore, the present study used KLF15-overexpressed TG mice to identify that the downregulated expression of KLF15 was not a consequence of myocardial interstitial remodeling, but it was a crucial factor in regulating pathways of anti-fibrosis or angiogenesis for preventing interstitial remodeling and HF (28).

However, these findings also raised some questions. First, AAC surgery was an acute coarctation process, which was different from the chronic development of rheumatic aortic stenosis. In the clinical practice, the compensatory period of cardiac function in patients with LV pressure overload was much longer than what was demonstrated in the present study. Additional delayed response factors may be involved in the LV remodeling process. Furthermore, it was still not clear whether the beneficial changes of protecting heart function were regulated by KLF15 in myocardiocytes independently or by a complex cross-talk between the non-myocardiocytes and myocardiocytes. Future studies would clarify this by establishing the co-culture system of myocardiocytes and non-myocardiocytes in vitro. Overall, KLF15 may be helpful in determining the optimum surgical timing and the evaluation of prognosis. Exogenous administration of KLF15 may play an important role in delaying heart failure in patients with LV overload disease in clinical treatment.

HF induced by pressure overload was revealed to be closely associated with myocardial interstitial remodeling, and KLF15 was the critical factor regulating the expression of CTGF, VEGF, p-p38 and p-Smad3 to effectively alleviate the progression of $\mathrm{HF}$ through repression of interstitial remodeling and the promotion of angiogenesis.

\section{Acknowledgements}

Not applicable.

\section{Funding}

The present study was supported by The National Natural Science Foundation of China (grant no. 81170216).

\section{Availability of data and materials}

The datasets used and/or analyzed during the current study are available from the corresponding author on reasonable request.

\section{Authors' contributions}

SFZ performed most of the experiments and wrote the manuscript. LC conceived the project. YBX and YP supervised the experiments and made substantial contributions to conception and design. YY, ZJ, YHJ, SC and FQT offered assistance with the data analysis and interpretation and manuscript revision.

\section{Ethics approval and consent to participate}

All procedures abided by the Guide for the Care and Use of Laboratory Animals (Department of Health and Human Services publication no. NIH 78-23,1996) and were approved by the Committee on Animal Research of Army Military Medical University, Chongqing, China.

\section{Patient consent for publication}

Not applicable.

\section{Competing interests}

The authors declare that they have no competing interests. 


\section{References}

1. Akinkuolie AO, Aleardi M, Ashaye AO, Gaziano JM and Djousse L: Height and risk of heart failure in the Physicians' Health Study. Am J Cardiol 109: 994-997, 2012.

2. Xu R, Lin F, Zhang S, Chen X, Hu S and Zheng Z: Signal pathways involved in reverse remodeling of the hypertrophic rat heart after pressure unloading. Int J Cardiol 143: 414-423, 2010.

3. Christia P, Bujak M, Gonzalez-Quesada C, Chen W, Dobaczewski M, Reddy A and Frangogiannis NG: Systematic characterization of myocardial inflammation, repair, and remodeling in a mouse model of reperfused myocardial infarction. J Histochem Cytochem 61: 555-570, 2013.

4. Ruwhof C and van der Laarse A: Mechanical stress-induced cardiac hypertrophy: Mechanisms and signal transduction pathways. Cardiovasc Res 47: 23-37, 2000.

5. Bernardo BC, Weeks KL, Pretorius L and McMullen JR: Molecular distinction between physiological and pathological cardiac hypertrophy: Experimental findings and therapeutic strategies. Pharmacol Ther 128: 191-227, 2010.

6. Berk BC, Fujiwara K and Lehoux S: ECM remodeling in hypertensive heart disease. J Clin Invest 117: 568-575, 2007.

7. Baudino TA, Carver W, Giles W and Borg TK: Cardiac fibroblasts: Friend or foe? Am J Physiol Heart Circ Physiol 291: H1015-H1026, 2006.

8. Leenders JJ, Wijnen WJ, van der Made I, Hiller M, Swinnen M, Vandendriessche T, Chuah M, Pinto YM and Creemers EE: Repression of cardiac hypertrophy by KLF15: Underlying mechanisms and therapeutic implications. PLoS One 7: e36754, 2012.

9. Yu Y, Ma J, Xiao Y, Yang Q, Kang H, Zhen J, Yu L and Chen L: KLF15 is an essential negative regulatory factor for the cardiac remodeling response to pressure overload. Cardiology 130 143-152, 2015.

10. Oka T, Akazawa H, Naito AT and Komuro I: Angiogenesis and cardiac hypertrophy: Maintenance of cardiac function and causative roles in heart failure. Circ Res 114: 565-571, 2014

11. Fisch S, Gray S, Heymans S, Haldar SM, Wang B, Pfister O, Cui L, Kumar A, Lin Z, Sen-Banerjee S, et al: Kruppel-like factor 15 is a regulator of cardiomyocyte hypertrophy. Proc Natl Acad Sci USA 104: 7074-7079, 2007.

12. Shindo T, Manabe I, Fukushima Y, Tobe K, Aizawa K, Miyamoto S, Kawai-Kowase K, Moriyama N, Imai Y, Kawakami H, et al: Kruppel-like zinc-finger transcription factor KLF5/BTEB2 is a target for angiotensin II signaling and an essential regulator of cardiovascular remodeling. Nat Med 8 : 856-863, 2002.

13. Chin MT: KLF15 and cardiac fibrosis: The heart thickens. J Mol Cell Cardiol 45: 165-167, 2008

14. Haldar SM, Lu Y, Jeyaraj D, Kawanami D, Cui Y, Eapen SJ, Hao C, Li Y, Doughman YQ, Watanabe M, et al: Klf15 deficiency is a molecular link between heart failure and aortic aneurysm formation. Sci Transl Med 2: 26ra26, 2010.

15. Yu Y, Zou S, Yang Q, Xiao Y, Yan Y, Chen H, Wu H, Luo Y, Yu P and Chen L: KLF15 is a positive regulatory factor in the process of myocardial remodeling and angiogenesis induced by pressure overload. Int J Clin Exp Med 9: 13394-13406, 2016.
16. Simoes PA, Zamarioli A, Bloes P, Mazzocato FC, Pereira LH, Volpon JB and Shimano AC: Effect of treadmill exercise on lumbar vertebrae in ovariectomized rats: Anthropometrical and mechanical analyses. Acta Bioeng Biomech 10: 39-41, 2008.

17. Karamanolis G, Delladetsima I, Kouloulias V, Papaxoinis K, Panayiotides I, Haldeopoulos D, Triantafyllou K, Kelekis N and Ladas SD: Increased expression of VEGF and CD31 in postradiation rectal tissue: Implications for radiation proctitis. Mediators Inflamm 2013: 515048, 2013.

18. Yan L, Wei X, Tang QZ, Feng J, Zhang Y, Liu C, Bian ZY, Zhang LF, Chen M, Bai X, et al: Cardiac-specific mindin overexpression attenuates cardiac hypertrophy via blocking AKT/GSK $3 \beta$ and TGF- $\beta 1-$ Smad signalling. Cardiovasc Res 92: 85-94, 2011.

19. Wynn TA and Ramalingam TR: Mechanisms of fibrosis: Therapeutic translation for fibrotic disease. Nat Med 18: 1028-1040, 2012.

20. Koitabashi N, Danner T, Zaiman AL, Pinto YM, Rowell J, Mankowski J, Zhang D, Nakamura T, Takimoto E and Kass DA: Pivotal role of cardiomyocyte TGF- $\beta$ signaling in the murine pathological response to sustained pressure overload. J Clin Invest 121: 2301-2312, 2011.

21. Teekakirikul P, Eminaga S, Toka O, Alcalai R, Wang L, Wakimoto H, Nayor M, Konno T, Gorham JM, Wolf CM et al: Cardiac fibrosis in mice with hypertrophic cardiomyopathy is mediated by non-myocyte proliferation and requires Tgf- $\beta$. J Clin Invest 120: 3520-3529, 2010.

22. Holzem KM, Marmerstein JT, Madden EJ and Efimov IR: Diet-induced obesity promotes altered remodeling and exacerbated cardiac hypertrophy following pressure overload. Physiol Rep 3: pii: e12489, 2015.

23. Pelouch V, Dixon IM, Golfman L, Beamish RE and Dhalla NS: Role of extracellular matrix proteins in heart function. Mol Cell Biochem 129: 101-120, 1993.

24. Cosaceanu D, Budiu RA, Carapancea M, Castro J, Lewensohn R and Dricu A: Ionizing radiation activates IGF-1R triggering a cytoprotective signaling by interfering with Ku-DNA binding and by modulating Ku86 expression via a p38 kinase-dependent mechanism. Oncogene 26: 2423-2434, 2007.

25. Leenders JJ, Wijnen WJ, Hiller M, van der Made I, Lentink V, van Leeuwen RE, Herias V, Pokharel S, Heymans S, de Windt LJ, et al: Regulation of cardiac gene expression by KLF15, a repressor of myocardin activity. J Biol Chem 285: 27449-27456, 2010.

26. Small EM, Thatcher JE, Sutherland LB, Kinoshita H, Gerard RD, Richardson JA, Dimaio JM, Sadek H, Kuwahara K and Olson EN: Myocardin-related transcription factor-a controls myofibroblast activation and fibrosis in response to myocardial infarction. Circ Res 107: 294-304, 2010.

27. Flanders KC, Major CD, Arabshahi A, Aburime EE, Okada MH, Fujii M, Blalock TD, Schultz GS, Sowers A, Anzano MA, et al: Interference with transforming growth factor-beta/Smad3 signaling results in accelerated healing of wounds in previously irradiated skin. Am J Pathol 163: 2247-2257, 2003.

28. Tian Y and Morrisey EE: Importance of myocyte-nonmyocyte interactions in cardiac development and disease. Circ Res 110: 1023-1034, 2012. 